\title{
A Study on the Characteristics of Nantong Fine-Brush Woodcarving Based on CNC Engraving and Manual Engraving
}

\author{
Wei-xia GAO ${ }^{1, a}$, Zhi-hui $\mathrm{Wu}^{1, \mathrm{~b}}$ and Yu-zhong JIANG ${ }^{2, \mathrm{c}}$ \\ ${ }^{1}$ College of Furniture and Industrial Design, Nanjing Forestry University, No159 Longpan Road, \\ Nanjing, Jiangsu, China \\ ${ }^{2}$ Jiangsu Nantong Sculpture Art Institute, No765 East Nanhai Road, Nantong, Jiangsu, China \\ a1046978972@qq.com, bwzh550@sina.com, cjiang185yuzhong@126.com
}

Keywords: Nantong fine-brush woodcarving, CNC engraving, Manual engraving, Characteristics. Abstract. Nantong fine-brush woodcarving is semi-processed first through CNC engraving machines which use Chinese traditional fine-brush paintings as carving patterns, then finished by manual engraving with hand and power tools. This paper dealt with the characteristics of Nantong fine-brush woodcarving from development background, the similarities and differences between it and Chinese traditional fine-brush painting, and main innovations compared with traditional bas-relief. This new woodcarving takes full advantages of numerically controlled machines and superb skills of engravers.

\section{Introduction}

Nantong fine-brush woodcarving is not only a new kind of woodcarving techniques based on Chinese traditional fine-brush painting and Chinese traditional bas-relief, but also a sort of products using the techniques. It uses red sanders, bois de rose, siam rosewood from the Chinese national standards "Hongmu" as main carved materials and uses the layout and modeling skills of Chinese traditional fine-brush painting as a reference. With the visual beauty from its unique theme, the texture and the technique, Nantong fine-brush woodcarving has greatly enriched artistic expression of bas-relief.

\section{The Development Background of Nantong Fine-Brush}

The fine brushwork is a special name of Chinese painting genre characterized by exquisiteness and neatness. As an important concept in the field of ancient Chinese painting, its painting image can be traced back to the Warring States silk painting "Human, Dragon and Phoenix Painting”, but the word "fine brushwork" first appeared in the documents of Northern Song Dynasty [1]. Since then, the word barely showed up until the Qing Dynasty. They had no unified name, although there were a lot of paintings during the past 600 years. The mature concept of fine brushwork was formed in the Qing Dynasty and widely spread. In this period, the fine brushwork was inclusive and communicative. As an adjective, it was combined into a compound word such as "fine brushwork plus theme" and "fine brushwork plus technique". Besides that, its concept was not limited to painting field, which was merged with other more popular art forms. Hence, the name fine-brush woodcarving came which was formed by the combination of fine brushwork and woodcarving.

Nantong fine-brush woodcarving is an attempt of bas-relief transformation launched by Nantong engravers from tradition to modernization in recent years. These people focus on how to develop the Chinese traditional bas-relief under the multicultural background. Despite its short development time as a new art form, its origins have a long and rich history. In fact, Chinese painting had been applied to woodcarving in the Ming and Qing Dynasties. For example, the panel of red sanders bed paneled with nanmu collected by the Qing palace, as shown in Fig. 1, was embossed with patterns of farming, fishery, landscape and figure. 


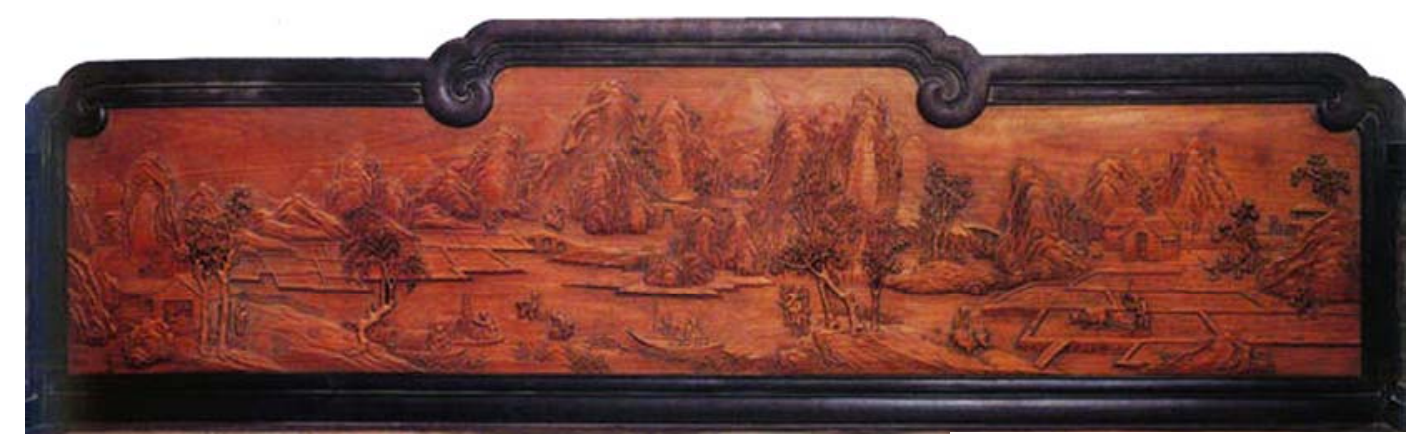

Fig. 1 The panel of red sanders bed paneled with nanmu collected by the Qing palace

\section{The Similarities and Differences between Nantong Fine-Brush Woodcarving and Chinese Traditional Fine-Brush Painting}

Nantong fine-brush woodcarving and Chinese traditional fine-brush painting belong to two different art forms, painting and sculpture, but they both are rooted in Chinese traditional culture and the former is developed on the basis of the latter. Therefore, there are some similarities and differences between them.

The Similar Subjects. The themes of Chinese traditional fine-brush painting can be divided into three main categories: landscape painting, figure painting and bird-and-flower painting [2]. It is evident that Nantong fine-brush woodcarving applies bird-and-flower theme to itself. Nantong fine-brush woodcarving is especially fond of the elegant literator's taste of flowers and birds, melons and fruits, insects and fishes because of its special regional culture [3]. Compared with the painting, Nantong fine-brush woodcarving pays more attention to the theme with well meanings and tends to choose images made up of plants and animals used as a homophone, metaphor or symbol. As shown in Fig. 2, the character ribbon of Asian Paradise-flycatcher is homophonic to character longevity in Chinese and the peony flower, which means blessing and richness.

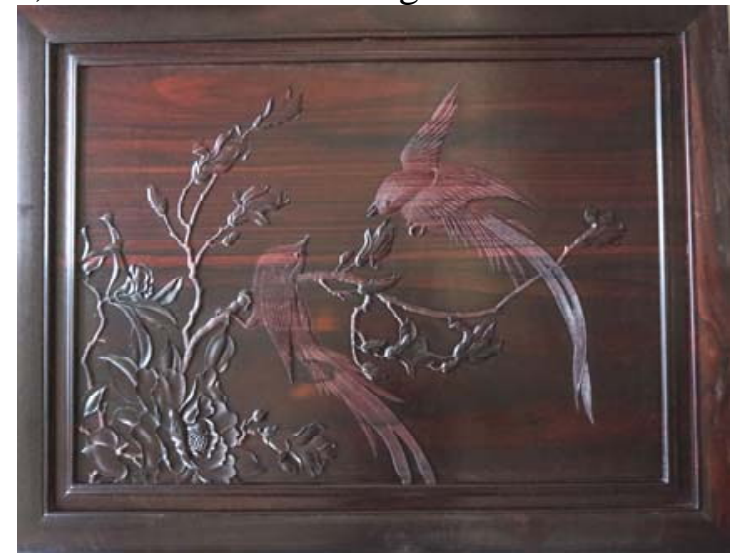

Fig. 2 The image combined with Asian Paradise-flycatcher, peony flower and magnolia of Nantong fine-brush woodcarving

The Different Materials and Tools. The materials and tools used in Nantong fine-brush woodcarving and Chinese traditional fine-brush painting vary greatly. The first is that the former uses the wood species of "Hongmu" as base materials, but the latter uses Xuan paper or silk paper. The second is that the former uses writing brush, ink, Chinese inkstand, mineral pigments as major tools, but the latter uses artisanal gravers. The sculptors usually use small electric routers and hand-held electric carving machines in comparison with painters using common hand tools, so that they could save time and effort to spend more energy on the finishing carving.

The Different Purposes of Techniques. There are many factors affecting the formation of the two different techniques in which, the most important is the differences between their purposes. Through the use of writing brush, ink, mineral pigments, Chinese traditional fine-brush painting adds materials 
on a piece of Xuan paper or silk paper, but Nantong fine-brush woodcarving uses gravers to remove material itself.

\section{The Main Innovations Compared with Traditional Bas-relief}

The Painterly Subjects. Nantong fine-brush woodcarving is different from Chinese traditional bas-relief. It mainly lies in that the former absorbs characteristics of Chinese traditional fine-brush painting such as scattering perspective, line modelling, image and artistic conception, which unlike Chinese traditional patterns used in the latter. As shown in Fig. 3, Nantong fine-brush woodcarving is also known as a woodcarving painting because it is appreciated by users and viewers in a two-dimensional plane from one direction and has much in common with Chinese traditional fine-brush painting [4].

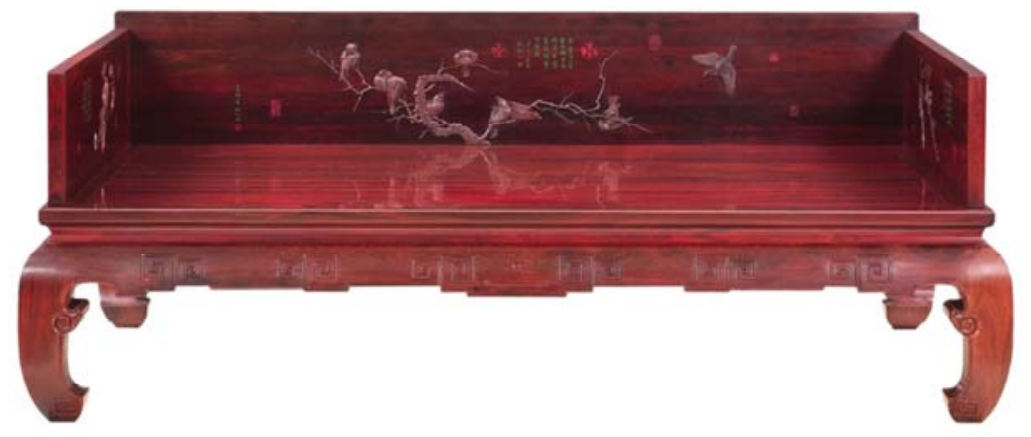

Fig. 3 The Lohan bed carved with trees and birds using the Nantong fine-brush woodcarving

The Innovative Techniques. The carving techniques are the methods of shape and space used by engravers. Nantong fine-brush woodcarving includes both traditional skills such as carving, engraving, shoveling and fine trimming and new innovative techniques which includes feathering, stipple engraving, texturing method. The innovative techniques rely on different engraving tools to implement themselves. As shown in Fig. 4, the cut of triangle gravers used for feathering is V-shaped. The furry animals of different shapes and sizes use different sizes of triangle gravers shown in Fig. 5. The so-called stipple engraving, which is relative to the line carving, is a discontinuous carving method. The texturing method is created to express different texture of objects. The latter two new techniques are mainly used in the tree barks and stones.
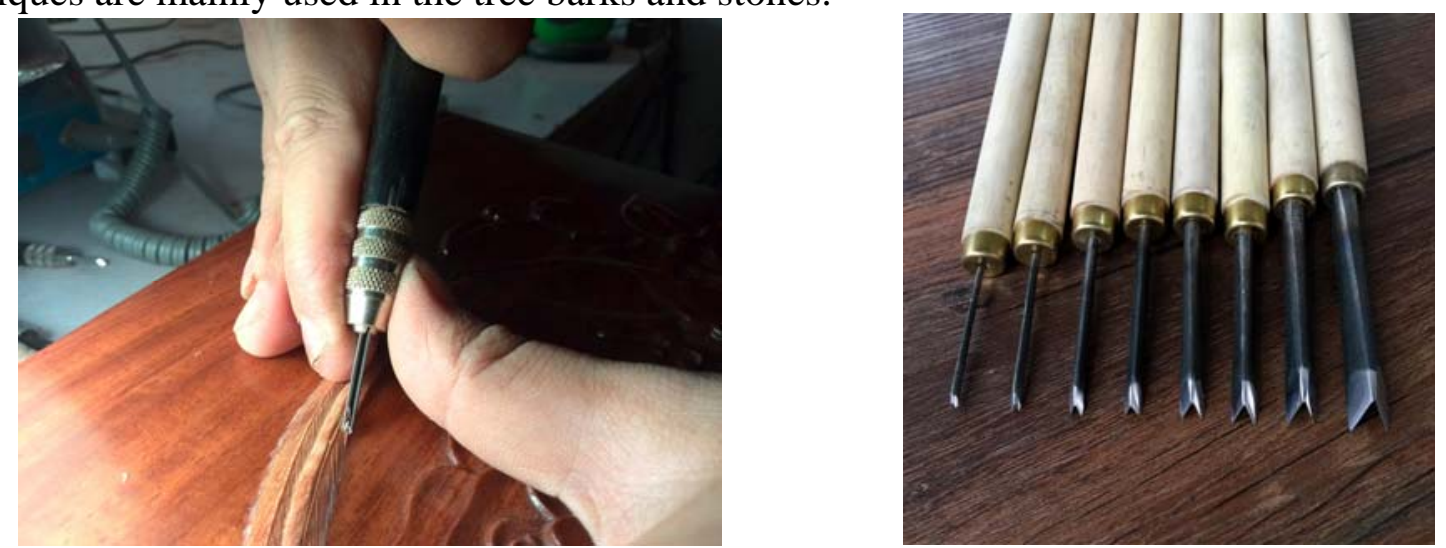

Fig. 4 The triangle graver used for carving feathers Fig. 5 The different sizes of triangle gravers

The Realistic-looking Textures. The texture in the art field refers to tactile texture and visual texture formed of the combination and treatment of materials [5]. For the Nantong fine-brush woodcarving, its formal beauty is the real texture which is the first thing that catches people's attention. The presented image texture is actually derived from natural texture. The engravers integrate visual feelings gained from the natural world into carving language, so the image texture is not a realistic representation but rather an aesthetic re-creation [6]. 
The images of different animals and plants in works of woodcarving play on contrasts in texture. For instance, the petals are nearly smooth, but the tree bark is rough, as shown in Fig. 6. Furthermore, there are differences of textures in different parts of one animal. Taking the bird covered with feathers as an example, the shapes and textures are not the same because of different body structures. As shown in Fig. 7, the head feathers are short and hard different from the soft belly feathers. In summary, Nantong fine-brush woodcarving creates a bird image through the textural effect of feathers, beaks, eyes and claws. The creation of textures has inconsistency and contingency due to the different intensities, directions and mentalities in the carving process. It will create a different texture effect even if using the same techniques, the same tools and the same material. According to the actual situation, people working as good engravers are very knowledgeable about the body structure, living habits, postures and expressions of animals and plants.

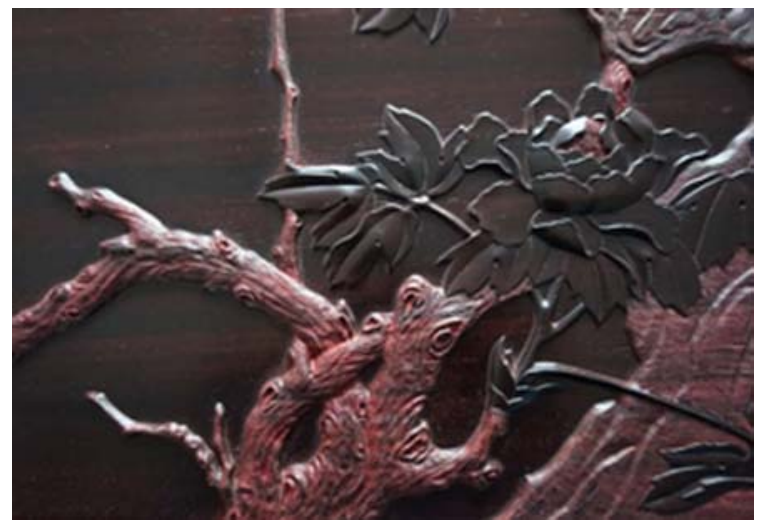

Fig. 6 The textures of petals and tree barks

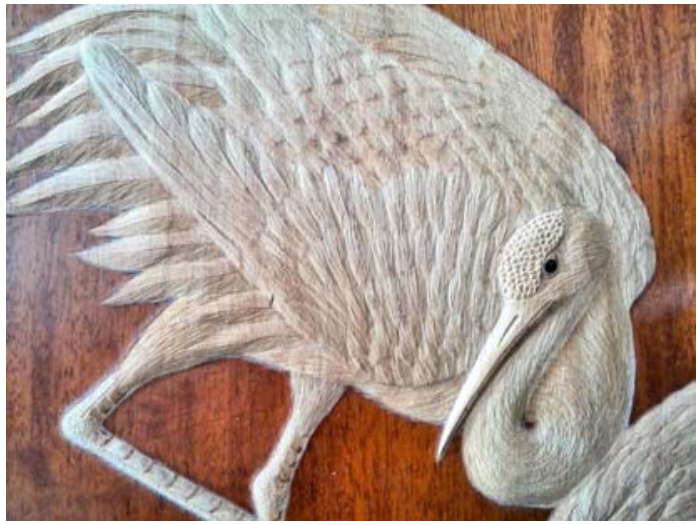

Fig. 7 The crane with different carved feathers

\section{Summary}

Nantong fine-brush woodcarving is a new bas-relief form, the most representative bas-relief form in Jiangsu and Zhejiang areas, based on the unique geographical environment, custom and culture of Jiangnan. It has been widely used in Chinese traditional Su-style furniture and handicrafts to increase their additional value and highly praised and appreciated by consumers.

\section{Acknowledgements}

This work was financially supported by the Priority Academic Program Development of Jiangsu Higher Education Institutions (PAPD).

\section{References}

[1] Niu Kecheng: A study on the origins of Chinese traditional fine-brush painting, Art. Vol. 12 (2013), p. 108-112.

[2] Zeng Jingxiang: Theoretical research on the techniques of Chinese traditional fine-brush painting (Hunan Arts Publications, China 2002).

[3] Chen Haibing: Interpretation on regional theme in regional painting, Hundred Schools in Arts. Vol. 8 (2012), p. 245-246.

[4] Shao Xiaofeng: An analysis of texture manufacture in the Chinese contemporary fine-brush painting (Nanjing Forestry University, Nanjing 2005).

[5] Bian Xiaoyan: The relationship between Chinese traditional furniture and painting (Yangzhou University, Yangzhou 2005).

[6] Li Min: On texture production in modern painting (Hangzhou Normal University, Hangzhou 2012). 